ARTIGO ESPECIAL / SPECIAL ARTICLE

\title{
Coorte retrospectiva de crianças e adolescentes hospitalizados por COVID-19 no Brasil do início da pandemia a 10 de agosto de 2020
}

\author{
Retrospective cohort of children and adolescents hospitalized by COVID-19 \\ in Brazil from the beginning of the pandemic to August 1 ${ }^{\text {st }}, 2020$
}

\author{
Nivreanes Tcherno Nulle Gomes' (D), Maria Isabella Claudino Haslett' (D), Ana Julia Silva e \\ Alves' (D), Jadher Percio' (D), Magda Machado Saraiva Duarte' $\mathbb{D}$, Juliane Maria Alves Siqueira \\ Malta' (1), Felipe Cotrim de Carvalho" (D), Walquiria Aparecida Ferreira de Almeida" (D), \\ Caroline Gava" (D), Libia Roberta de Oliveira Souza" (D), Francieli Fontana Sutile \\ Tardetti Fantinato" (D), Elizabeth David dos Santos'
}

\begin{abstract}
RESUMO: Objetivos: Caracterizar a população do estudo, estimar a taxa de letalidade intra-hospitalar por estado e analisar fatores associados aos óbitos por COVID-19. Métodos: Foi realizado estudo de coorte retrospectiva de crianças e adolescentes hospitalizados com diagnóstico de COVID-19 confirmado por transcrição reversa seguida de reação em cadeia da polimerase (RT-PCR), tendo como desfecho óbito por COVID-19 ou recuperação, entre $1^{\circ}$ de março e $1^{\circ}$ de agosto de 2020 . A fonte de dados foi o Sistema de Informação de Vigilância Epidemiológica da Gripe (SIVEP-Gripe), ao qual são notificados pacientes internados com Síndrome Respiratória Aguda Grave (SRAG). Consideraram-se crianças os pacientes com idade entre 0 e 11 anos completos e adolescentes aqueles com idade entre 12 e 18 anos completos. Realizou-se análise bi e multivariável por meio de Regressão de Poisson com variância robusta, utilizando-se como medida de associação final o Risco Relativo ajustado (RRa). Resultados: Dos 4.930 casos analisados, 2.553 (51,8\%) eram do sexo masculino. A raça/cor autodeclarada parda foi a mais frequente, com $2.335(47,4 \%)$. A unidade federativa de Roraima apresentou a maior taxa de letalidade intra-hospitalar, com $68,8 \%(\mathrm{n}=11 / 16)$. A análise multivariada mostrou que pertencer ao grupo etário adolescente $(\mathrm{RR}=1,59 ; \mathrm{IC} 95 \% 1,12-2,25 ; \mathrm{p}=0,009)$, ter sido classificado como SRAG-crítico $(\mathrm{RR}=$ 4,56; IC95\% 2,77 - 7,51; p < 0,001) e apresentar imunopatia (RR = 2,24; IC95\% 1,58 - 3,17; p < 0,001) como comorbidade configuraram-se como fatores associados ao óbito pela COVID-19. Conclusão: Observou-se que ser adolescente, ter classificação de SRAG-crítico e imunopatia como comorbidade foram importantes fatores associados ao óbito. Recomenda-se vigilância ativa e cuidados diferenciados a portadores de doenças crônicas e condições imunológicas especiais.
\end{abstract}

Palavras-chave: Criança. Adolescente. Hospitalização. Infecções por coronavírus. Síndrome respiratória aguda grave.

'Programa de Treinamento em Epidemiologia Aplicada aos Serviços do Sistema Único de Saúde, Coordenação-Geral de Emergências em Saúde Pública, Departamento de Saúde Ambiental, do Trabalhador e Vigilância das Emergências em Saúde Pública, Secretaria de Vigilância em Saúde, Ministério da Saúde - Brasília (DF), Brasil.

"Coordenação-Geral do Programa Nacional de Imunizações, Departamento de Imunização e Doenças Transmissíveis, Secretaria de Vigilância em Saúde, Ministério da Saúde - Brasília (DF), Brasil.

Autor correspondente: Nivreanes Tcherno Nulle Gomes. QMSW 5, Lote 3, Bloco H, ap. 207, Setor Sudoeste, CEP: 70680-500, Brasília, DF, Brasil. E-mail: nullegomes@gmail.com

Conflito de interesses: nada a declarar - Fonte de financiamento: Programa de Treinamento Epidemiologia Aplicada aos Serviços do Sistema Único de Saúde, da Secretaria de Vigilância em Saúde do Ministério da Saúde, e Projeto Regional de Reforço dos Sistemas de Vigilância das Doenças em África Ocidental (REDISSE). 
ABSTRACT: Objectives: To characterize the study population, estimating the in-hospital lethality rate by state and analysing associated factors with COVID-19-related deaths. Methods: A retrospective cohort study was carried out of hospitalised children and adolescents diagnosed with COVID-19, confirmed by RT-PCR, whose outcome was death by COVID-19 or recovery, from 2020 March 1 to August 1. The data source was the Influenza Epidemiological Surveillance Information System (SIVEP-Gripe in Brazilian acronym), where patients with Severe Acute Respiratory Syndrome (SARS) are notified. Children were defined as those between the ages of 0 and 11, and adolescents those between 12 and 18. A bi and multivariate analysis were performed using Poisson Regression with robust variance, with adjusted Relative Risk as the final association measure. Results: A total of 4,930 cases were analysed; 2,553 (51.8\%) were males, 2,335 (47.4\%) were brown-skinned. The Federative Unit of Roraima presented the highest in-hospital case-fatality rate, with $68.8 \%(11 / 16)$. Multivariate analysis showed that belonging to the age group adolescent $(R R=1.59 ; 95 \% C I 1.12-2.25 ; \mathrm{p}=0.009)$, SARS-critical patient $(\mathrm{RR}=4.56$; $95 \% \mathrm{CI} 2,77-7.51 ; \mathrm{p}<0.001)$ and presenting immunological disorders $(\mathrm{RR}=2.24 ; 95 \% \mathrm{CI} 1.58-3.17 ; \mathrm{p}<0.001)$ as comorbidities were statistically associated factors with death by COVID-19. Conclusion: It was observed that adolescents, SARS-critical patients, and presence of immunological disorders were important factors associated with death. Active surveillance and differentiated care are recommended for patients with chronic diseases and special immunological conditions.

Keywords: Child. Adolescent. Hospitalization. Coronavirus infections. Severe acute respiratory syndrome.

\section{INTRODUÇÃO}

Em dezembro de 2019 o mundo se colocou em estado de alerta, quando um novo tipo de coronavírus (denominado posteriormente de SARS-CoV-2) foi descoberto por afetar o trato respiratório de humanos, causando desde infecções assintomáticas às manifestações mais graves, com síndromes respiratórias agudas que podem evoluir para óbito. Os primeiros casos, que tiveram origem em Wuhan, na China, propagaram-se rapidamente pelo território chinês, atingindo todo o continente asiático e expandindo-se para os continentes europeu e americano em menos de três meses ${ }^{1-3}$, até que, em 30 de janeiro de 2020, a Organização Mundial da Saúde (OMS) declarou que a dispersão dos casos da COVID-19 pelo mundo constituía-se numa Emergência de Saúde Pública de Importância Internacional (ESPII)².

A transmissão do vírus ocorre por meio do contato com gotículas respiratórias geradas por pessoas infectadas. As manifestações diferenciam-se de organismo para organismo, a depender do sistema imunológico, das condições preexistentes, da idade e da quantidade de vírus com a qual se teve contato ${ }^{4}$.

Crianças e adolescentes parecem ter sido menos afetados pela pandemia, já que neles a infecção ocorre de maneira mais leve $e^{5}$. Mais de um mês após o início dos casos, em 20 de janeiro de 2020, a China notificou o primeiro caso em criança. Estudos realizados na China, em países Europeus e nos Estados Unidos da América estimam que de 1 a 5\% dos casos confirmados de COVID-19 ocorram no subgrupo populacional de crianças e adolescentes ${ }^{5,6}$. Ainda, o primeiro grande relatório do Centro de Controle e Prevenção de Doenças da China 
mostrou que, dos 44.672 casos confirmados, apenas uma morte ocorreu entre crianças e adolescentes ${ }^{7}$. Já no Brasil, a notificação do primeiro caso nesse grupo etário foi em 4 de março, cerca de uma semana após a notificação da chegada do vírus ao país ${ }^{8}$.

Até a semana epidemiológica (SE) 31, que teve fim no dia $1^{\circ}$ de agosto de 2020 , foram confirmados 17.580 .163 casos da doença em todo o mundo. Na ocasião, o Brasil já acumulava 2.707.877 casos $^{9}$.

Entre as questões que buscam explicar o número relativamente pequeno de crianças e adolescentes afetados pela COVID-19 estão o fato de essas pessoas apresentarem o desenvolvimento do sistema imunológico celular e humoral em curso, a possível proteção por infecções prévias pelo vírus sincicial respiratório e a imaturidade dos receptores chamados de enzimas conversoras da angiotensina 2 (ACE-2) na infância ${ }^{10}$. Além disso, destacam-se as medidas de controle propostas pelos governos, que incluem o fechamento das instituições de ensino e o consequente maior isolamento social ${ }^{11}$.

Um aspecto muito discutido refere-se ao papel das crianças e adolescentes como reservatórios do SARS-CoV-2 e na dinâmica de transmissão da doença ${ }^{12-16}$. A adoção de medidas preventivas como a etiqueta respiratória, a lavagem das mãos, o distanciamento social, entre outras, não é bem compreendida por esse grupo etário e, para as crianças menores de dois anos, pediatras recomendam a não utilização de máscaras, pois existe o risco de sufocamento ${ }^{17,18}$.

No fim do mês de abril de 2020, médicos do Reino Unido lançaram um alerta sobre a ocorrência de manifestações clínicas temporalmente relacionadas à infecção pela COVID-19 em crianças e adolescentes, denominada de Síndrome Inflamatória Multissistêmica Pediátrica ${ }^{19}$.

Diante dessas peculiaridades relacionadas a essas faixas etárias e da importância de se compreender o impacto do novo coronavírus nessa população, faz-se necessário gerar evidências sobre as hospitalizações de crianças e adolescentes pela COVID-19 no Brasil a fim de contribuir com a condução de ações que protejam e promovam a saúde desse público, bem como subsidiar a tomada de decisão.

Nesse sentido, este artigo tem como objetivo geral analisar as crianças e adolescentes hospitalizados por COVID-19 no Brasil, com os objetivos específicos de:

- caracterizar a população de estudo em tempo, pessoa e lugar;

- estimar a taxa de letalidade intra-hospitalar por unidade federativa (UF);

- analisar fatores associados aos óbitos por COVID-19 nesse subgrupo populacional.

\section{MÉTODOS}

Realizou-se um estudo de coorte retrospectiva de crianças e adolescentes hospitalizados, com diagnóstico de COVID-19 confirmado no teste de biologia molecular (transcrição reversa seguida de reação em cadeia da polimerase - RT-PCR em tempo real) e com resultado detectável para SARS-CoV-2, tendo como desfecho óbito por COVID-19 ou recuperação.

Foram utilizados dados secundários, não nominais, provenientes do Sistema de Informação de Vigilância Epidemiológica da Gripe (SIVEP-Gripe), de domínio público, disponibilizados 
no sítio eletrônico do Ministério da Saúde (MS) e acessados no dia 14 de setembro de 2020, incluindo os casos notificados com data de início de sinais e sintomas entre $1^{\circ}$ de março e $1^{\circ}$ de agosto de 2020 (SE 10 a 31).

Os dados analisados compreenderam as 27 UF do País, com população estimada de 211,7 milhões de pessoas em 2020. Os menores de 19 anos representam 31,2\%, com 66,1 milhões de crianças e adolescentes ${ }^{20}$.

Considerou-se como criança o indivíduo com idade entre 0 (zero) e 11 anos completos e adolescente aquele pertencente à faixa etária de 12 a 18 anos completos ${ }^{21}$.

Foram utilizadas as seguintes definições de caso:

- Síndrome Respiratória Aguda Grave (SRAG): caso de COVID-19 com a presença de pelo menos um dos seguintes sinais e sintomas: dispneia, desconforto respiratório, baixa saturação de O2 (<95\%) em ar ambiente e cianose $\mathrm{e}^{22}$.

- SRAG-crítico: caso de SRAG que necessitou de internação em Unidade de Terapia Intensiva (UTI) ou precisou de suporte ventilatório, invasivo ou não invasivo.

A definição de SRAG foi baseada naquela descrita no capítulo de Vigilância de Síndromes Respiratórias Agudas do Guia de Vigilância Epidemiológica ${ }^{22}$, e a definição de SRAG-crítico elaborada para este estudo foi adaptada das classificações de Chung et al. ${ }^{23}$, a fim de analisar o nível de gravidade e o fator de risco para o óbito.

As variáveis de interesse abordadas neste estudo foram: semana epidemiológica de início dos primeiros sintomas; evolução (óbitos e recuperados); data de evolução; UF de residência e de internação; sexo (masculino, feminino e indeterminado); idade em anos; raça/cor autodeclarada (branca, preta, amarela, parda e indígena); sinais e sintomas; comorbidade; resultados de Raio X de tórax (normal, infiltrado intersticial, consolidação, misto e outros); internação em UTI; suporte ventilatório (invasivo e não invasivo); e uso de antiviral. Para a análise de fatores associados ao óbito, foram criadas e acrescentadas variáveis secundárias com base nas principais, a saber: idade em anos/grupo etário (criança e adolescente); raça/ cor (preta/parda e branca) $)^{24,25}$; SRAG e SRAG-crítico.

Para a análise descritiva foram calculadas medidas de frequência absoluta e relativa, de tendência central e dispersão. A taxa de letalidade intra-hospitalar foi calculada considerando-se o número total dos óbitos hospitalares dividido pelo total de casos hospitalizados, multiplicado por 100. Para a análise bivariada, aplicou-se o teste de hipóteses Exato de Fisher, como medida de associação o Risco Relativo bruto (RR) com intervalo de confiança (IC) de $95 \%$ e p $<0,05$, e, na comparação entre medianas, utilizou-se o teste não paramétrico de Mann-Whitney.

A fim de retirar possíveis fatores de confundimento, realizou-se análise estatística multivariada por meio de Regressão de Poisson com variância robusta, considerando-se o número de variáveis independentes obtidas pela análise bivariada $(\mathrm{p}<0,20)$. Sendo assim, optou-se pela realização de uma regressão do tipo backward stepwise. A medida de associação adotada foi o Risco Relativo ajustado (RR ajustado), respeitando-se a ausência de multicolinearidade (correlação entre duas ou mais variáveis independentes) no modelo. Foi considerado o teste de razão de verossimilhança para a escolha do melhor modelo. 
Para o processamento e a análise dos dados, foram utilizados os programas Epi InfoTM 7.2, Stata/SE 13.1, QGIS 2.18 “Las palmas” e Microsoft Excel 2016.

\section{RESULTADOS}

No período entre $1^{\circ}$ de março e $1^{\circ}$ de agosto de 2020, correspondente às SE 10 a 31 , foram notificados 302.066 casos hospitalizados por COVID-19, dos quais 295.867 (97,9\%) eram adultos e idosos e 6.199 (2,1\%) eram crianças e adolescentes. Destes, 365 (5,9\%) foram diagnosticados pelos critérios clínico e epidemiológico e 904 (14,6\%) não tinham registro do desfecho. Conforme demonstrado na Figura 1, no período do estudo, 4.930 (79,5\%) casos com desfecho (óbito ou recuperação) constituíram a população do estudo, dos quais 500 $(10,1 \%)$ evoluíram ao óbito e $4.430(89,9 \%)$ foram classificados como recuperados. Entre os óbitos, 323 (64,6\%) eram crianças (zero a 11 anos completos).

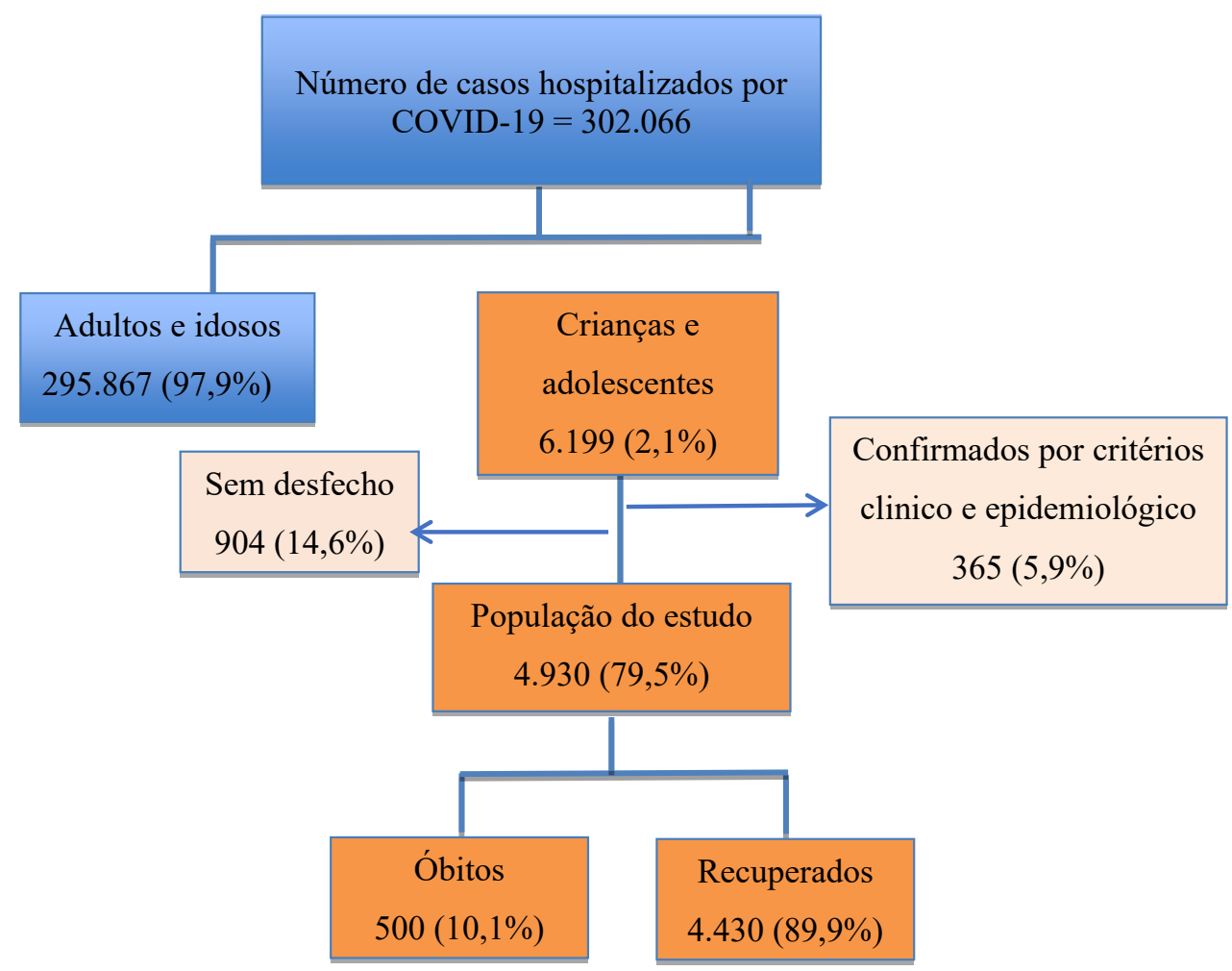

Fonte: Dados do SIVEP-Gripe atualizados até 14 de setembro de 2020.

Figura 1. Fluxograma de distribuição de casos hospitalizados por COVID-19, de $1^{\circ}$ março a $1^{\circ}$ agosto, Brasil, 2020. 
Com relação à distribuição dos casos ao longo do período, observou-se que 1.554 (31,5\%) das hospitalizações ocorreram entre as SE 10 a 20 ( $1^{\circ}$ de março a 16 de maio de 2020 ), com média de três óbitos / dia, e $3.376(68,5 \%)$ entre as SE 21 a 31 (17 de maio a $1^{\circ}$ de agosto de 2020), com média de quatro óbitos / dia (dados não apresentados em tabela).

Considerando-se a distribuição dos casos de acordo com a UF de residência, São Paulo teve a maior frequência de pacientes hospitalizados, com 1.320 (26,8\%), dos quais 1.247 $(94,5 \%)$ se recuperaram e 73 (4,5\%) evoluíram a óbito. Em seguida, vem o Amazonas com $517(10,5 \%)$ pacientes hospitalizados, dos quais $491(95,0 \%)$ se recuperaram e $26(5 \%)$ foram a óbito (Figuras 2A e 2B e Material Suplementar).

(A)

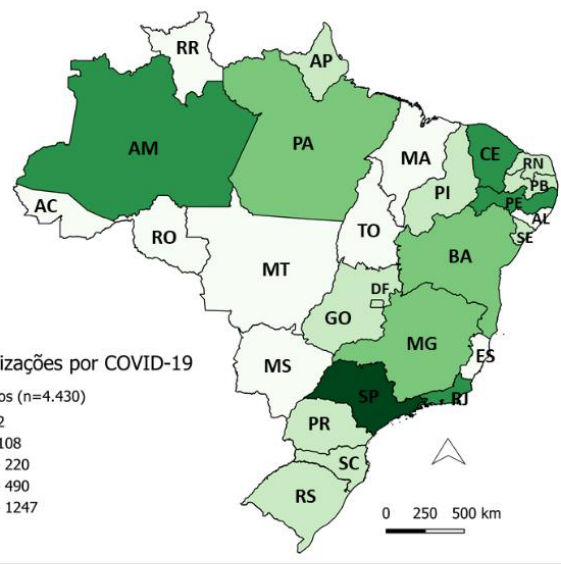

(B)

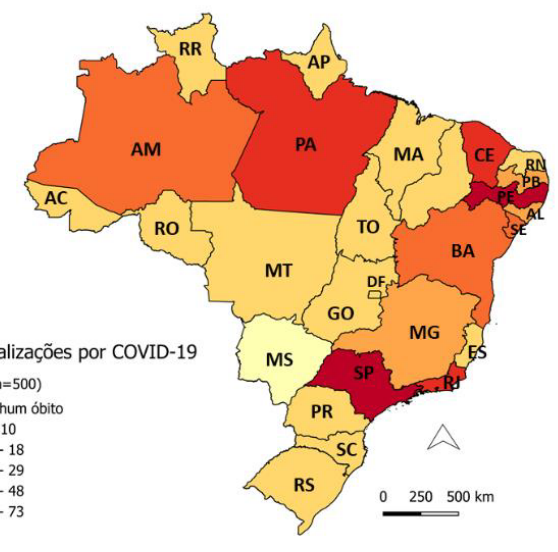

(C)

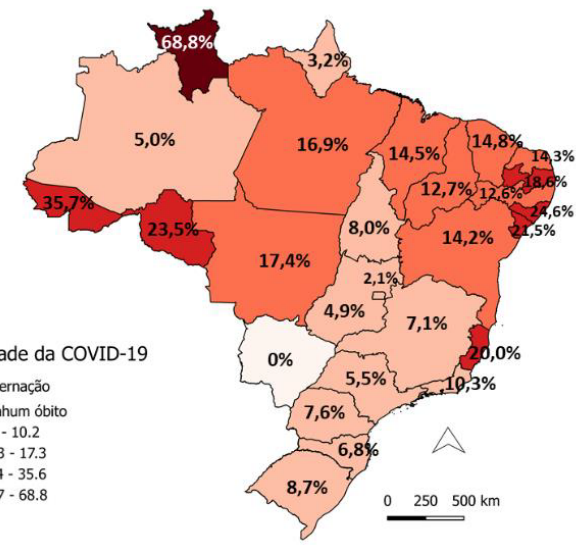

Fonte: Dados do SIVEP-Gripe atualizados até 14 de setembro de 2020.

Figura 2. (A) Casos recuperados de crianças e adolescentes hospitalizados por COVID-19 segundo unidade federativa de residência, $1^{\circ}$ de março a $1^{\circ}$ de agosto, Brasil, 2020; (B) Óbitos de crianças e adolescentes hospitalizados por COVID-19 segundo unidade federativa de residência, $1^{\circ} \mathrm{de}$ março a $1^{\circ}$ de agosto, Brasil, 2020; (C) Taxas de letalidade intra-hospitalar por unidade federativa de internação, $1^{\circ}$ de março a $1^{\circ}$ de agosto, Brasil, 2020. 
No que se refere às maiores taxas de letalidade intra-hospitalar por UF de internação, Roraima apresentou 68,8\% $(n=11 / 16)$, Acre 35,7\% $(n=5 / 14)$ e Alagoas $24,6 \%(n=17 / 69)$ (Figura $2 C)$.

Do total de 4.930 casos, 3.610 eram crianças $(73,2 \%)$ e $1.320(26,8 \%)$ adolescentes. O sexo masculino representou $2.553(51,8 \%)$.

A média de idade das crianças e adolescentes foi de 6,2 anos (DP =6,4). A média dos que evoluíram a óbito foi de 6,8 anos $(\mathrm{DP}=7,1)$ e, para os recuperados, foi de 6,1 anos $(\mathrm{DP}=6,4)$ (Tabela 1$)$.

As maiores taxas de letalidade ocorreram nas faixas etárias de 15 a 18 anos, com 13,6\%, de 12 a 14 anos, com 13,1\%, e de menores de um ano de idade, com 12,6\% (Tabela 1).

Entre os menores de um ano de idade, 171 (4,7\%) tiveram início de sintomas no mesmo dia de nascimento, dos quais 33 (19,3\%) evoluíram a óbito. Entre estes últimos, 18 (54,5\%) foram internados em UTI, com mediana de zero dia (Q1-Q3: 0 - 0) entre a data do início dos sinais

Tabela 1. Caracterização de casos hospitalizados por COVID-19 em crianças e adolescentes, segundo sexo, faixa etária, raça/cor e evolução $(n=4.930)$ de $1^{\circ}$ de março a $1^{\circ}$ de agosto, Brasil, 2020.

\begin{tabular}{|c|c|c|c|c|}
\hline \multirow{2}{*}{ Características } & \multicolumn{2}{|c|}{ Óbitos $(n=500)$} & \multicolumn{2}{|c|}{ Recuperados $(n=4.430)$} \\
\hline & $n$ & $\%$ & $\mathrm{n}$ & $\%$ \\
\hline \multicolumn{5}{|l|}{ Sexo } \\
\hline Masculino & 266 & 10,4 & 2.287 & 89,6 \\
\hline Feminino & 234 & 9,8 & 2.142 & 90,2 \\
\hline Indeterminado & 0 & 0,0 & 1 & 100,0 \\
\hline \multicolumn{5}{|l|}{ Faixa etária (anos) } \\
\hline$<1$ & 190 & 12,6 & 1.318 & 87,4 \\
\hline 1 a 4 & 67 & 6,0 & 1.048 & 94,0 \\
\hline 5 a 11 & 66 & 6,7 & 921 & 93,3 \\
\hline 12 a 14 & 54 & 13,1 & 359 & 86,9 \\
\hline 15 a 18 & 123 & 13,6 & 784 & 86,4 \\
\hline \multicolumn{5}{|c|}{ Raça/cor autodeclarada } \\
\hline Branca & 98 & 7,9 & 1.139 & 92,1 \\
\hline Preta & 14 & 10,4 & 121 & 89,6 \\
\hline Amarela & 2 & 8,0 & 23 & 92,0 \\
\hline Parda & 250 & 10,7 & 2.085 & 89,3 \\
\hline Indígena & 19 & 22,1 & 67 & 77,9 \\
\hline Sem informação & 117 & 10,5 & 995 & 89,5 \\
\hline Idade (anos) & \multicolumn{2}{|c|}{ Média } & \multicolumn{2}{|c|}{ Desvio Padrão } \\
\hline Todos os casos & \multicolumn{2}{|c|}{6,2} & \multicolumn{2}{|c|}{6,4} \\
\hline Óbitos & \multicolumn{2}{|c|}{6,8} & \multicolumn{2}{|c|}{7,1} \\
\hline Recuperados & \multicolumn{2}{|c|}{6,1} & \multicolumn{2}{|c|}{6,4} \\
\hline
\end{tabular}

Fonte: Dados do SIVEP-Gripe atualizados até 14 de setembro de 2020. 
e sintomas e a internação. A mediana entre a data do início dos sinais e sintomas e a data do diagnóstico por RT-PCR foi de seis dias (Q1-Q3: 3 - 9) (dados não apresentados em tabela).

Dos 4.930 casos, 2.335 (47,4\%) autodeclararam-se da raça/ cor parda, dos quais $250(10,7 \%)$ evoluíram a óbito. A raça/cor branca vem em seguida, com $1.237(25,1 \%)$ casos, dos quais $98(7,9 \%)$ foram a óbito (Tabela 1$)$.

O total de $1.219(24,7 \%)$ casos foi internado em UTI. Destes, 300 (24,6\%) evoluíram a óbito e 919 (75,4\%) recuperaram-se. Quanto aos exames de imagem dos casos que se internaram em UTI, $667(54,7 \%)$ tinham registro de radiografia de tórax, com resultado de infiltrado intersticial presente em 68 (22,7\%) óbitos e 187 (20,3\%) recuperados. No que se refere ao suporte ventilatório durante a internação em UTI, de 822 casos $(67,4 \%)$ que receberam esse recurso, $442(53,8 \%)$ necessitaram do tipo invasivo, dos quais $220(49,8 \%)$ foram a óbito e $222(50,2 \%)$ recuperaram-se (dados não apresentados em tabela).

Dos 4.930 casos, 3.180 (64,5\%) atenderam à definição de SRAG, entre os quais 1.857 $(37,7 \%)$ se enquadraram na definição de SRAG-crítico.

Os sinais e sintomas mais frequentes, entre os 500 casos de SRAG que evoluíram para óbito, foram dispneia com 350 (70\%), desconforto respiratório com 346 (69,2\%), febre com $339(67,8 \%)$ e baixa saturação de oxigênio com 300 (60\%) (Figura 3A). Entre os 207 óbitos com registro de comorbidades, as mais frequentes foram a imunopatia com $56(27,1 \%)$, a cardiopatia com $53(25,6 \%)$ e a neuropatia com 50 (24,2\%) (Figura 3B).

A mediana de tempo entre o início dos sintomas e a hospitalização foi de três dias tanto para os óbitos como para os recuperados (Q1-Q3: 0-6 e 1-6, respectivamente; $\mathrm{p}=0,006$ ); A mediana de tempo entre a internação e a evolução, para os que foram a óbito, foi de seis dias (Q1-Q3: 2 - 14) e, para os recuperados, de cinco dias (Q1-Q3: 3 - 11) com valor de $\mathrm{p}=0,971$; e, entre a internação em UTI e a evolução, a mediana foi de cinco dias tanto para os óbitos quanto para os recuperados (Q1-Q3: 2 - 14 e $2-11$ respectivamente; $\mathrm{p}=0,591$ ).

$\mathrm{Na}$ análise bivariada, observou-se que tiveram maior risco de evoluir ao óbito os casos que apresentaram os seguintes fatores: ser do grupo etário de adolescentes (RR = 1,49; IC95\% $1,26$ - 1,78; $\mathrm{p}<0,001)$; ser da raça/ cor autodeclarada preta/parda (RR = 1,34; IC95\% 1,08 - 1,68; $\mathrm{p}<0,008$ ); ter sido classificado como SRAG-crítico (RR = 4,13; IC95\% 3,43 - 3,96; $\mathrm{p}<0,001)$; ter cardiopatia ( $R R=2,07$; IC95\% 1,58 - 2,72; $\mathrm{p}<0,001)$, imunopatia $(\mathrm{RR}=1,74$; IC95\% 1,32-2,30; $\mathrm{p}<0,001)$, diabetes $(R R=1,57$; IC95\% 1,08-2,30; $p=0,032)$ e neuropatia $(\mathrm{RR}=1,47 ; \mathrm{IC} 95 \% 1,09-1,97 ; \mathrm{p}=0,013)$. A asma configurou-se como fator de redução do risco para o óbito $(R R=0,25 ;$ IC95\% 0,10-0,62; $\mathrm{p}=0,003$ (Tabela 2).

Outras variáveis relacionadas aos sinais e sintomas respiratórios foram testadas na análise bivariada, quais sejam: baixa saturação de oxigênio ( RR = 4,12; IC95\% 3,33 - 5,09; p < 0,001), desconforto respiratório ( $R R=3,23$; I95\% 2,57 - 4,06; $\mathrm{p}<0,001)$, dispneia $(\mathrm{RR}=3$; IC95\% $2,39-3,78 ; \mathrm{p}<0,001)$ e cianose $(\mathrm{RR}=2,89$; IC95\% 1,66 - 5,05; $\mathrm{p}=0,003$ ) (dados não apresentados em tabela). Essas variáveis foram agrupadas no conjunto da variável SRAG-crítico para compor o melhor modelo de regressão.

A análise multivariada mostrou que permaneceram como fatores associados ao óbito pela COVID-19: pertencer ao grupo etário adolescente $(R R=1,59 ;$ IC95\% 1,12-2,25; $\mathrm{p}=0,009)$, ter sido classificado como SRAG-crítico (RR = 4,56; IC95\% 2,77-7,51; $<<0,001)$ e apresentar

\section{8}

REV BRAS EPIDEMIOL 2021; 24: E210026 
(A)

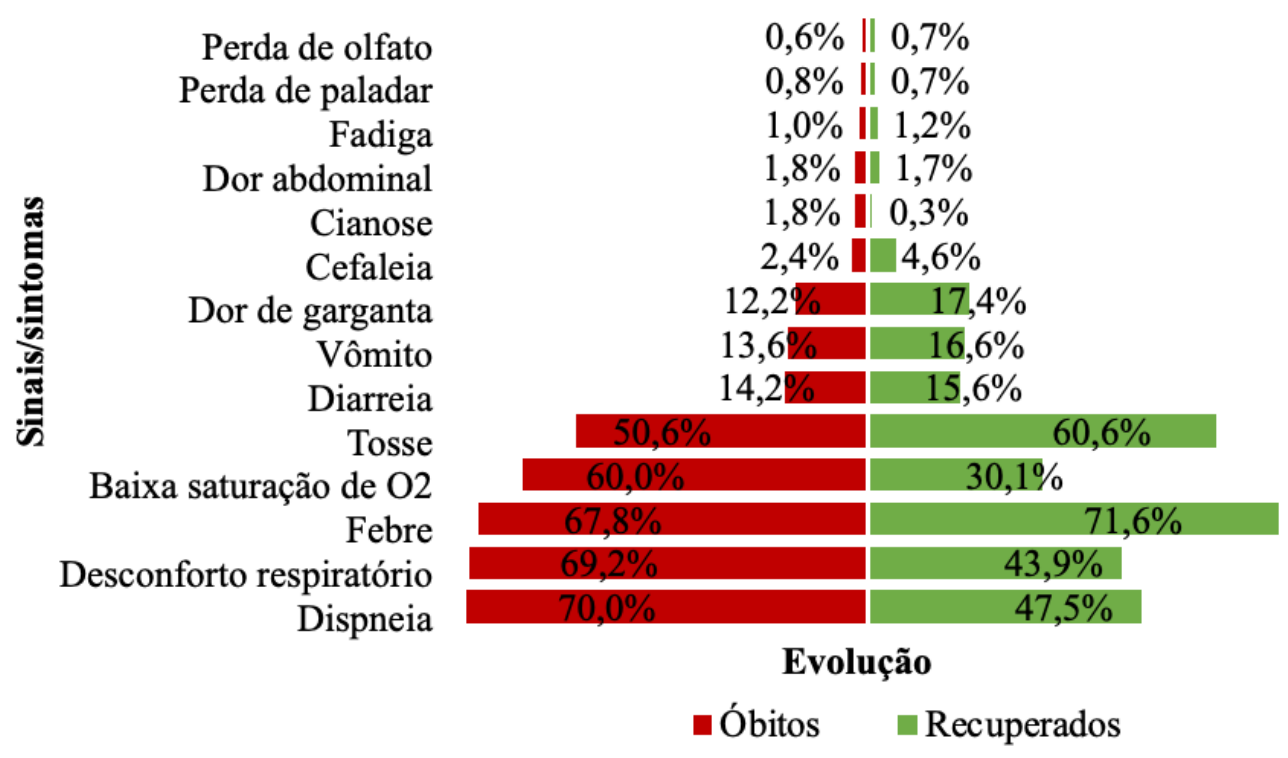

(B)

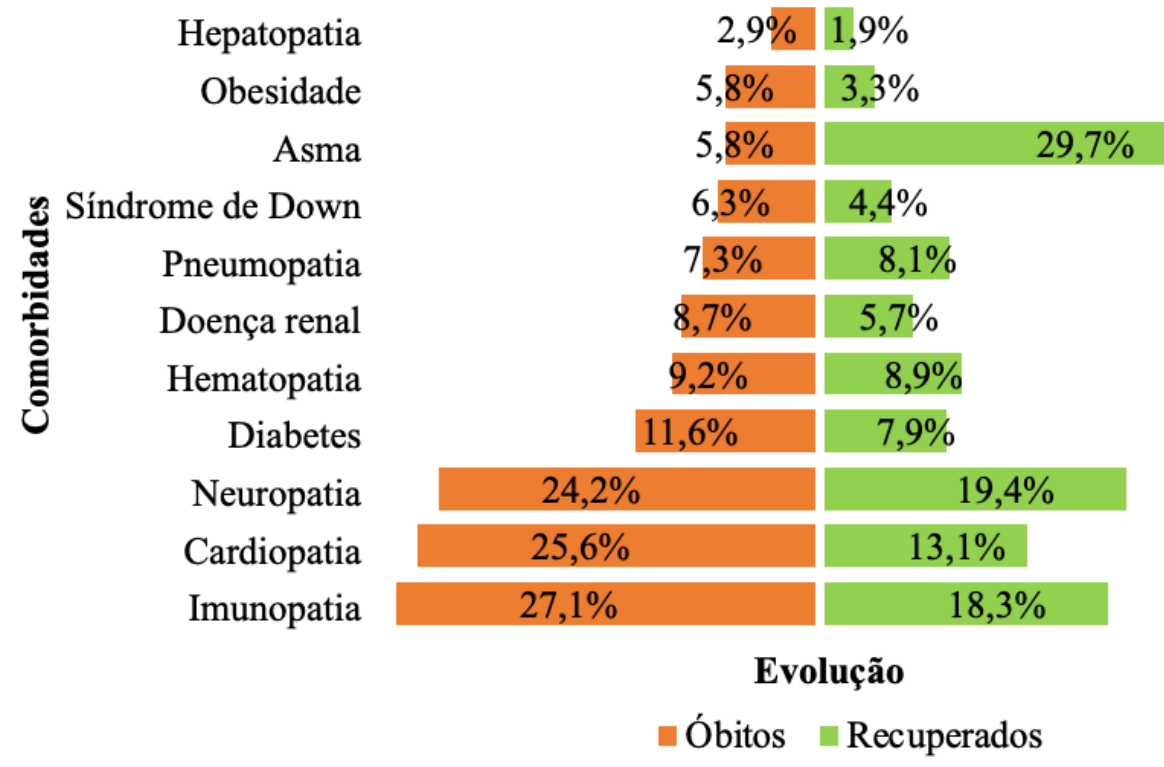

Fonte: Dados do SIVEP-Gripe atualizados até 14 de setembro de 2020.

Figura 3. (A) Sinais e sintomas dos casos hospitalizados por COVID-19 em crianças e adolescentes segundo evolução, $1^{\circ}$ de março a $1^{\circ}$ de agosto, Brasil, 2020 ( $n$ = 4.930); (B) Comorbidades dos casos hospitalizados por COVID-19 em crianças e adolescentes segundo evolução, $1^{\circ}$ de março a $1^{\circ}$ de agosto, Brasil, $2020(n=1.214)$. 
Tabela 2. Análise bivariada e multivariada de fatores associados ao risco de evoluir ao óbito por COVID-19 em crianças e adolescentes, $1^{\circ}$ de março a $1^{\circ}$ de agosto, Brasil, $2020\left(R^{2}=0,1381\right)$.

\begin{tabular}{l|c|c|c|c|c|c}
\hline \multirow{2}{*}{ Variáveis } & \multicolumn{3}{|c|}{ RR Bruto } & \multicolumn{3}{c}{ RR Ajustado } \\
\cline { 2 - 7 } & RR & IC95\% & Valor $p$ & RRa & IC95\% & Valor $p$ \\
\hline Grupo etário/adolescente & 1,49 & $1,26-1,78$ & $<0,001$ & 1,59 & $1,12-2,25$ & 0,009 \\
\hline Raça/cor preta/parda & 1,34 & $1,08-1,68$ & 0,008 & - & - & - \\
\hline Tosse & 0,81 & $0,67-0,98$ & 0,035 & 0,78 & $0,55-1,10$ & 0,162 \\
\hline Cefaleia & 0,37 & $0,27-0,81$ & 0,003 & 0,38 & $0,09-1,49$ & 0,167 \\
\hline Dor de garganta & 0,79 & $0,60-1,03$ & 0,084 & - & - & - \\
\hline SRAG-crítico & 4,13 & $3,43-4,97$ & $<0,001$ & 4,56 & $2,77-7,51$ & $<0,001$ \\
\hline Cardiopatia & 2,07 & $1,58-2,72$ & $<0,001$ & 1,49 & $0,94-2,37$ & 0,086 \\
\hline Neuropatia & 1,47 & $1,09-1,97$ & 0,013 & 1,45 & $0,92-2,27$ & 0,107 \\
\hline Imunopatia & 1,74 & $1,32-2,30$ & $<0,001$ & 2,24 & $1,58-3,17$ & $<0,001$ \\
\hline Diabetes & 1,57 & $1,08-2,30$ & 0,032 & - & - & - \\
\hline Doença renal & 1,64 & $1,07-2,51$ & 0,044 & - & - & - \\
\hline Asma & 0,22 & $0,12-0,39$ & $<0,001$ & $\mathbf{0 , 2 5}$ & $\mathbf{0 , 1 0 - \mathbf { 0 , 6 2 }}$ & $\mathbf{0 , 0 0 3}$ \\
\hline Obesidade & 1,81 & $1,09-3,00$ & 0,035 & - & - & - \\
\hline Síndrome de Down & 1,57 & $0,95-2,58$ & 0,124 & - & - & - \\
\hline Uso de antiviral & 1,16 & $0,93-1,45$ & 0,180 & - & - & -
\end{tabular}

RR: risco relativo; IC95\%; intervalo de confiança de 95\%; RRa: risco relativo ajustado.

Fonte: Dados do SIVEP-Gripe atualizados até 14 de setembro de 2020.

imunopatia $(\mathrm{RR}=2,24 ; \mathrm{IC} 95 \% 1,58-3,17 ; \mathrm{p}<0,001)$. A asma permaneceu como fator associado à redução do risco para o óbito $(\mathrm{RR}=2,24 ; \mathrm{IC} 95 \%$ 1,58 - 3,17; $\mathrm{p}<0,001)$ (Tabela 2).

\section{DISCUSSÃO}

O presente estudo caracterizou os casos de COVID-19 ocorridos em crianças e adolescentes no Brasil que foram hospitalizados e notificados no SIVEP-Gripe. Mais de dois terços dos casos foram registrados em menores de cinco anos e mais da metade dos casos ocorreu em crianças e adolescentes do sexo masculino.

Embora a população mais suscetível ao novo coronavírus seja composta principalmente por idosos com baixa imunidade ${ }^{26}$, destaca-se que os vírus respiratórios são uma causa comum de infecção do trato respiratório em crianças, sendo considerados importante motivo de internações ${ }^{27}$.

No começo da pandemia, iniciou-se a discussão sobre o papel das crianças e adolescentes como reservatórios do SARS-CoV-2 e na dinâmica de transmissão da doença ${ }^{12-16}$. É importante ressaltar que esses cenários mudaram com as novas apresentações clínicas da doença em crianças. Isso porque algumas delas evoluíram para a Síndrome Inflamatória 
Multissistêmica Pediátrica, com manifestações clínicas graves e quadro semelhante aos observados em crianças e adolescentes com síndrome de Kawasaki, Kawasaki incompleto e/ou síndrome do choque tóxico ${ }^{28}$, os quais não foram objetos deste estudo.

Verificou-se que um número considerável de crianças apresentou sinais e sintomas com menos de 24 horas do nascimento e que quase um quinto dessas crianças evoluíram a óbito, a maioria tendo sido internada em UTI no mesmo dia em que nasceu - embora ainda faltem informações sobre a condição sorológica das mães durante a gestação. Mesmo assim, levanta-se a suspeita de transmissão vertical, corroborando Alzamora et al., que relataram casos de infecção em recém-nascidos com RT-PCR positivo entre 16 e 30 horas após o nascimento, apontando para essa possibilidade ${ }^{29}$.

A raça/cor autodeclarada parda representa metade dos que evoluíram ao óbito, tratando-se da raça/cor predominante na população brasileira de baixa renda, ao lado da preta ${ }^{25}$, o que indica a importância das desigualdades sociais na evolução da doença. O estudo de Silva Filho et al. sugere que a carência da infraestrutura domiciliar leva a maior risco de contágio e propagação de infecções respiratórias ${ }^{30}$. Souza mostrou ainda que a mortalidade por doenças do aparelho respiratório aumentou de forma preocupante em todas as regiões do Brasil $^{31}$. Diante dos considerados níveis de desigualdade social no país, é provável um efeito desproporcional da COVID-19 entre os mais vulneráveis.

No que se refere aos sinais e sintomas apresentados, a maioria dos que evoluíram a óbito apresentou dispneia, desconforto respiratório, febre, baixa saturação de oxigênio e tosse. Quanto às comorbidades, houve predomínio de imunopatias, seguido de cardiopatias não especificadas e neuropatias.

Um estudo realizado no Irã com 30 crianças hospitalizadas com COVID-19 encontrou resultados semelhantes em relação às manifestações clínicas e comorbidades. Houve relato de leucemia entre as comorbidades presentes em algumas crianças que evoluíram ao óbito, sendo febre, dispneia e tosse os sinais e sintomas mais frequentemente apresentados ${ }^{32}$.

Quase um terço dos hospitalizados necessitaram de cuidados em UTI. Porém, mais de um terço dos que precisaram de suporte ventilatório o receberam de forma invasiva. Entre as alterações observadas na radiografia de tórax desses pacientes, mais de dois terços dos que apresentaram infiltrado intersticial foram a óbito.

A UF de Roraima apresentou a maior taxa de letalidade intra-hospitalar no período analisado. Essa taxa pode estar superestimada, pois essa UF e o Acre, juntamente, apresentam os menores registros de hospitalização, o que pode sugerir subnotificação dos casos hospitalizados. Cabe recordar que, para este estudo, foram considerados somente os casos com desfecho (óbito ou recuperação).

A análise multivariada dos fatores associados ao óbito por COVID-19 mostrou que os adolescentes apresentaram maior risco de evoluir a óbito em relação às crianças. Algumas hipóteses podem explicar tal peculiaridade, entre elas a de que as crianças apresentam menor expressão da ECA-2, e os receptores ECA-2 imaturos na infância podem dificultar a invasão celular. A outra seria a existência de alguma proteção em razão de infecções anteriores pelo vírus sincicial respiratório ${ }^{10,33}$. Os que evoluíram para a fase crítica da doença (SRAG-crítico) apresentaram quase cinco vezes mais risco de evoluir para óbito. A tosse e a cefaleia não 
foram fatores diretamente associados, mas foram determinantes para o aumento do risco quando presentes, ajustando de maneira independente com outras variáveis o modelo de regressão analisado.

Entre as comorbidades, a imunopatia foi fator importante associado ao óbito por COVID19 em crianças e adolescentes hospitalizados. Neste estudo, observou-se que, apesar de grande parte dos que evoluíram a óbito terem apresentado cardiopatia e/ou neuropatia como doença de base, essas comorbidades não permaneceram como fatores diretamente associados ao óbito, tendo sido, porém, importantes para o aumento de risco, ajustando de forma independente com outras variáveis o modelo de regressão analisado.

Considerando-se que a COVID-19 afeta predominantemente o trato respiratório e que as infeções por vírus respiratórios são causas frequentes de complicação da asma, existe a preocupação de que a COVID-19 tenha apresentação mais grave em doentes asmáticos. Dessa forma, várias instituições ligadas à saúde consideraram os doentes com asma como provável grupo de risco para COVID-1934,35. Neste estudo, a asma foi observada como fator de redução de risco para o óbito por COVID-19 na análise bivariada e multivariada. É possível que aqueles que têm essa doença de base estivessem controlados no momento que foram infectados por SARS-CoV-2 ou tenham recebido cuidados diferenciados durante a internação. Também pode ser que estivessem sob tratamento com corticosteroide antes de se infectarem com SARS-CoV-2, o que pode influenciar positivamente na evolução da COVID-19 $9^{36}$.

Destaca-se que os resultados deste estudo representam a população de crianças e adolescentes hospitalizados por COVID-19 e notificados no SIVEP-Gripe em todo o território nacional. Entre as limitações, não foi possível analisar as variáveis tiragem intercostal e batimento de asa nasal, que são sinais de gravidade importantes em criança por serem variáveis não existentes no banco, e esses dados foram registrados facultativamente como outros sinais. Ainda, muitos sinais e sintomas podem estar subestimados em razão da alta ocorrência de incompletude, decorrente da falta de atualização do banco de acordo com a evolução do paciente.

É importante destacar que as crianças e adolescentes, além de serem vistos como grupo etário com importante papel na dinâmica de transmissão ${ }^{12-16}$, também apresentam fatores de vulnerabilidade para agravamento. Portanto, realça-se que ainda há muito a se descobrir sobre a infecção pelo SARS-CoV-2 em crianças e adolescentes. Entretanto, os achados deste estudo fazem refletir sobre os subgrupos mais vulneráveis e as condições imunológicas relacionadas à gravidade e ao óbito.

É importante que crianças e adolescentes vulneráveis à gravidade e ao óbito pela COVID19 sejam incluídos nas políticas públicas de saúde como grupo de risco. Além disso, é necessário, durante a pandemia, manter a vigilância ativa para crianças e adolescentes, principalmente as portadoras de doenças crônicas, sobretudo imunopatias, cardiopatias e neuropatias, as quais fazem parte de um grupo de grande importância e de maior risco para a gravidade e óbito pela COVID-19. Ademais, é importante realizar investigação detalhada das crianças diagnosticadas com COVID-19 que tiveram início de sinais e sintomas em menos de 24 horas do nascimento, para evidenciar uma possível transmissão vertical. 


\section{REFERÊNCIAS}

1. Kamps BS, Hoffmann C. COVID Reference [Internet]. Hamburg: Steinhäuser Verlag; 2020 [acessado em 11 abr. 2020]. Disponível em: https: / / covidreference.com

2. Zhu N, Zhang D, Wang W, Li X, Yang B, Song J, et al. A novel coronavirus from patients with pneumonia in China, 2019. N Engl J Med 2020; 382(8): 727-33.

3. . Pan American Health Organization, World Health Organization. Brasil. Folha Informativa. COVID-19 (Doença Causada Pelo Novo Coronavírus) [Internet]. Pan American Health Organization, World Health Organization; 2020 [acessado em 11 abr. 2020]. Disponível em: https://www.paho.org/bra/index.php

4. Saxena SK, editor. Coronavirus Disease 2019 (COVID19): Epidemiology, Pathogenesis, Diagnosis, and Therapeutics. Springer Nature; 2020.

5. CDC COVID-19 Response Team. Coronavirus Disease 2019 in Children - United States, February 12-April 2, 2020. MMWR Morb Mortal Wkly Rep 2020; 69(14): 422-6. https: / / doi.org/10.15585/ mmwr.mm6914e4

6. Bellino S, Punzo O, Rota MC, Manso M, Urdiales AM, Andrianou X, et al. COVID-19 Disease Severity Risk Factors for Pediatric Patients in Italy. Pediatrics 2020; 146(4): e2020009399. https: / / doi.org/10.1542/ peds.2020-009399

7. Safadi MA. The intriguing features of COVID-19 in children and its impact on the pandemic. J Pediatr (Rio J) 2020; 96(3): 265-8. https: / / doi.org/10.1016/j. jped.2020.04.001

8. Brasil. Ministério da Saúde. Secretaria de Vigilância em Saúde. Coordenação-Geral do Programa Nacional de Imunizações. Grupo Técnico-Influenza. Dados do Sivep Gripe. Brasil: Ministério da Saúde; 2020.

9. Brasil. Boletim Epidemiológico COVID-19. 2020; (25).

10. Nunes MDR, Pacheco STA, Costa CIA, Silva JA, Xavier WS, Victória JZ. Exames diagnósticos e manifestações clínicas da COVID-19 em crianças: revisão integrativa. Texto Contexto - Enferm 2020; 29: e20200156. https: / / doi.org/10.1590/1980-265x-tce-2020-0156

11. Vilelas JMDS. O novo coronavírus e o risco para a saúde das crianças. Rev Latino-Am Enfermagem 2020; 28. https: / / doi.org/10.1590/1518-8345.0000.3320

12. Lu X, Zhang L, Du H, ZhangJ, Li YY, Qu J, et al. SARSCoV-2 infection in children. N Engl J Med 2020; 382(17): 1663-5. https: / / doi.org/10.1056/nejmc2005073

13. Qiu H, Wu J, Hong L, Luo Y, Song Q, Chen D. Clinical and epidemiological features of 36 children with coronavirus disease 2019 (COVID-19) in Zhejiang, China: an observational cohort study. Lancet Infect Dis 2020; 20(6): 689-96. http://dx.doi.org/10.1016/ S1473-3099(20)30198-5
14. Zou L, Ruan F, Huang M, Liang L, Huang H, Hong $Z$, et al. SARS-CoV-2 viral load in upper respiratory specimens of infected patients. N Engl J Med 2020; 382(12): 1177-9. https:/ / doi.org/10.1056/nejmc2001737

15. Kam K, Yung CF, Cui L, Pin Lin RT, Mak TM, Maiwald $\mathrm{M}$, et al. A well infant with coronavirus disease 2019 (COVID-19) with high viral load. Clin Infect Dis 2020; 71(15): 847-9. http: / / dx.doi.org/10.1093/ cid/ ciaa201

16. Zhang T, Cui X, Zhao X, Wang J, Zheng J, Zheng G, et al. Detectable SARS-CoV-2 viral RNA in feces of three children during recovery period of COVID-19 Pneumonia. J Med Virol 2020; 92(7): 909-14. http:/ / dx.doi.org/10.1002/jmv.25795

17. Zomer TP, Erasmus V, van Beeck EF, Tjon-A-Tsien A, Richardus JH, Voeten HA. Hand hygiene compliance and environmental determinants in child day care centers: an observational study. Am J Infect Control 2013; 41(6): 497-502. https://doi.org/10.1016/j. ajic.2012.06.005

18. Clark J, HenkJK, Crandall PG, Crandall MA, O’Bryan CA. An observational study of handwashing compliance in a child care facility. Am J Infect Control 2016; 44(12): 1469-74. https:/ / doi.org/10.1016/j.ajic.2016.08.006

19. Davies P, Evans C, Kanthimathinathan HK, Lillie J, Brierley J, Waters G, et al. Intensive care admissions of children with paediatric inflammatory multisystem syndrome temporally associated with SARS-CoV-2 (PIMS-TS) in the UK: a multicentre observational study. Lancet Child Adolesc Health 2020; 4: 669-77. https: / / doi.org/10.1016/S2352-4642(20)30215-7

20. Instituto Brasileiro de Geografia e Estatística. Estratificação da população estimada segundo faixas etárias [Internet]. Brasil: Instituto Brasileiro de Geografia e Estatística [acessado em 21 maio 2020]. Disponível em https: / observatoriocrianca.org.br/ cenario-infancia/temas/populacao/1048-estratificacaoda-populacao-estimada-pelo-ibge-segundo-faixasetarias?filters $=1,1626 ; 1,1627$

21. Brasil. Presidência da República. Lei ${ }^{0} 8.609$ de 13 de julho de 1990-Estatuto da Criança e do Adolescente [Internet]. 1990 [acessado em $1^{\circ}$ out. 2020]. Disponível em: http:/ / www.planalto.gov.br/ccivil_03/leis/18069.htm

22. Brasil. Ministério da Saúde. Secretaria de Vigilância em Saúde. Coordenação-Geral do Programa Nacional de Imunizações. Grupo Técnico-Influenza. Guia de Vigilância Epidemiológica. Vigilância de Síndromes Respiratórias Agudas. COVID-19. Brasil: Ministério da Saúde; 2020.

23. Chung M, Bernheim A, Mei X, Zhang N, Huang M, Zeng X, et al. CT imaging features of 2019 novel 
coronavirus (2019-nCoV). Radiology 2020; 295(1): 202-7. https: / / doi.org/10.1148/radiol.2020200230

24. Instituto Brasileiro de Geografia e Estatística. Diretoria de Pesquisas. Coordenação de Trabalho e Rendimento. Pesquisa Nacional por Amostra de Domicílio Contínua 2012/2019 [Internet]. Instituto Brasileiro de Geografia e Estatística [acessado em 22 maio 2020]. Disponível em https: / / www.ibge.gov.br/estatisticas/sociais / trabalho/17270-pnad-continua.html? = \&t=o-que-e

25. Instituto Brasileiro de Geografia e Estatística. Diretoria de Pesquisas. Coordenação de População e Indicadores Sociais. Desigualdades sociais por cor ou raça no Brasil [Internet]. Rio de Janeiro: Instituto Brasileiro de Geografia e Estatística; 2019 [acessado em 22 maio 2020]. Disponível em: https: / / biblioteca.ibge.gov.br/index. $\mathrm{php} /$ biblioteca catalogo?view $=$ detalhes\&id $=2101681$

26. Li W, Cui H, Li K, Fang Y, Li S. Chest computed tomography in children with COVID-19 respiratory infection. Pediatr Radiol [Internet] 2020 [acessado em 14 abr. 2020]; 50(6): 796-9. Disponível em: https:// dx.doi.org/10.1007/s00247-020-04656-7

27. Lin C-Y, Hwang D, Chiu N-C, Weng L-C, Liu H-F, Mu $\mathrm{J}-\mathrm{J}$, et al. Increased detection of viruses in children with respiratory tract infection using PCR. Int J Environ Res Public Health [Internet]. 2020 [acessado em 11 abr. 2020]; 17(2): 564. Disponível em: https: / / dx.doi. org/10.3390/ijerph17020564

28. Mahase E. Covid-19: concerns grow over inflammatory syndrome emerging in children. BMJ [Internet] 2020 [acessado em 13 jun. 2020]; 369: m1710. Disponível em: https:/ / dx.doi.org/10.1136/bmj.m1710

29. Alzamora MC, Paredes T, Caceres D, Webb CM, Valdez LM, Rosa M. COVID-19 grave durante a gravidez e possível transmissão vertical. Am J Perinatol 2020; 37(8): 861-5. https: / / doi.org/10.1055/ s-0040-1710050

30. Silva Filho EBS, Silva AL, Santos AO, Dall'Acqua DSV, Souza LFB. Infecções Respiratórias de Importância Clínica: uma Revisão Sistemática. Rev FIMCA 2017; 4(1): 716. https: / / doi.org/10.37157/fimca.v4i1.5

31. Souza IDTD. Mortalidade por doenças respiratórias no Brasil e suas regiões: série histórica 2000-2013 [trabalho de conclusão de curso]. Natal: Universidade Federal do Rio Grande do Norte; 2016.

32. Soltani J, Sedighi I, Shalchi Z, Sami G, Moradveisi B, Nahidi S. Pediatric coronavirus disease 2019 (COVID-19): An insight from west of Iran. North Clin Istanb [Internet] 2020 [acessado em 11 jun. 2020]; 7(3): 284-91. Disponível em: https:/ / dx.doi. org/10.14744\%2Fnci.2020.90277
33. Sociedade Brasileira de Pediatria. Departamento Científico de Pneumologia. Nota de Alerta. COVID-19 em crianças: envolvimento respiratório [Internet]. Sociedade Brasileira de Pediatria; 2020 [acessado em 13 jun. 2020]. Disponível em: https: / / www.sbp.com.br/imprensa/detalhe/nid/ covid-19-em-criancas-envolvimento-respiratorio/

34. Portugal. Direção-Geral de Saúde. COVID-19: Fase de mitigação abordagem do doente com suspeita ou infeção por SARS-CoV-2. Norma Orientação Clínica 004/2020 [Internet]. 2020 [acessado em 30 jul. 2020]. Disponível em: https:// covid19.minsaude.pt/normas

35. Center of Disease Control COVID-19 Response Team. Preliminary estimates of the prevalence of selected underlying health conditions among patients with coronavirus disease 2019 [Internet]. MMWR Morb Mortal Wkly Rep 2020 [acessado em 30 jul. 2020]; 69(13): 382-6. Disponível em: https: / /www.cdc.gov/mmwr/volumes/69/wr/ pdfs/mm6913e2-H.pdf

36. The WHO Rapid Evidence Appraisal for COVID19 Therapies (REACT) Working Group. Association Between Administration of Systemic Corticosteroids and Mortality Among Critically Ill Patients With COVID-19. JAMA 2020; 324(13): 1330-41. http:/ / doi. org/10.1001/jama.2020.17023

\section{Recebido em: 01/03/2021 \\ Revisado em: 14/04/2021 \\ Aceito em: 14/04/2021 \\ Preprint em: 16/04/2021}

Contribuições dos autores: Gomes, NTN; Haslett, MIC; Santos, ED: concepção e delineamento do estudo, análise e discussão dos resultados, revisão e aprovação da versão final do artigo. Duarte, MMS: concepção e delineamento do estudo, revisão e aprovação da versão final do artigo. Percio, J; Alves, AJS; Malta, JMAS: delineamento do estudo, discussão dos resultados, revisão e aprovação da versão do artigo. Carvalho, FC; Almeida, WAF; Gava, C; Souza, LRO; Fantinato, FFST: interpretação dos dados, revisão e aprovação da versão do artigo. Todos os autores concordam e são responsáveis por todos os aspectos do trabalho, no sentido de garantir que as questões relacionadas à exatidão ou à integridade de qualquer parte da obra sejam investigadas e resolvidas. 


\section{ERRATA / ERRATUM}

No artigo "Coorte retrospectiva de crianças e adolescentes hospitalizados por COVID-19 no Brasil do início da pandemia a $1^{\circ}$ de agosto de 2020”, DOI: 10.1590/1980-549720200026, publicado no periódico Rev Bras Epidemiol. 2021; 23: e210026:

\section{Página 5, Figura 1:}

Onde se lia:

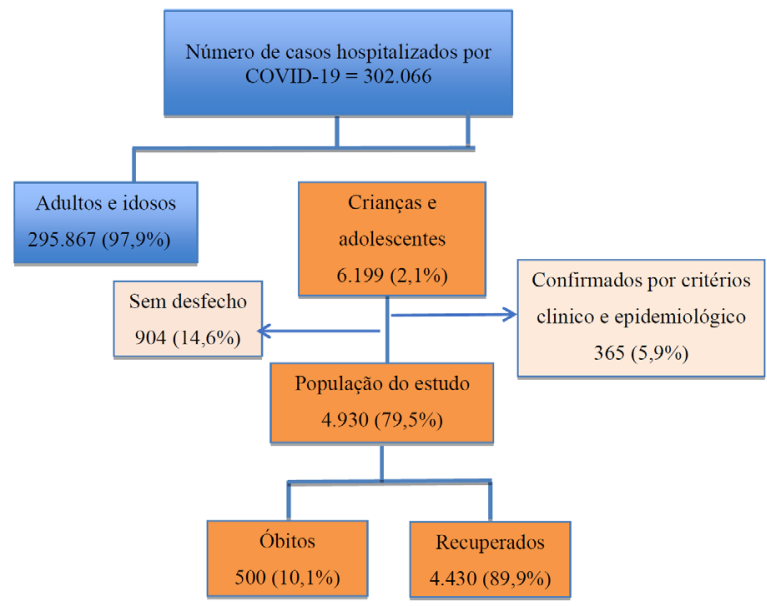

Fonte: Dados do SIVEP-Gripe atualizados até 14 de setembro de 2020.

Figura 1. Fluxograma de distribuição de casos hospitalizados por COVID-19, de $1^{0}$ de março a $1^{\circ}$ de agosto. Brasil, 2020.

\section{Leia-se:}

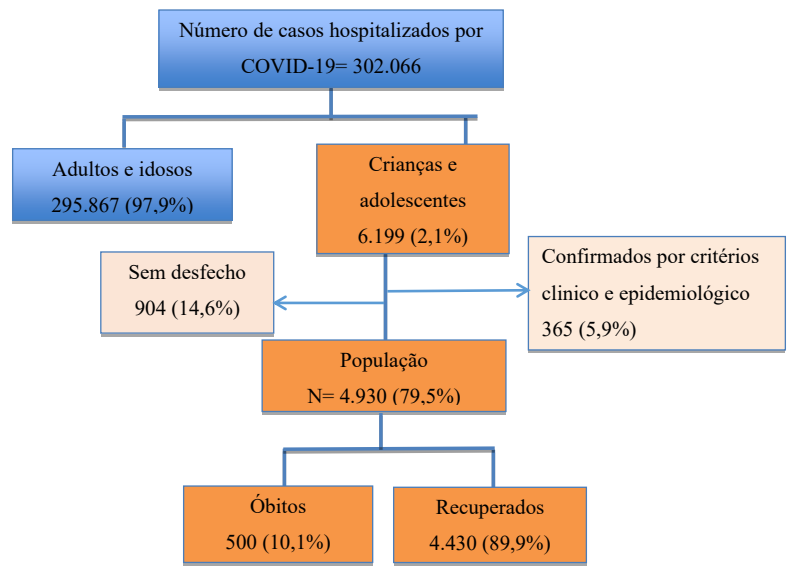

Fonte: Dados do SIVEP-Gripe atualizados até 14 de setembro de 2020.

Figura 1. Fluxograma de distribuição de casos hospitalizados por COVID-19, de $1^{0}$ de março a $1^{\circ}$ de agosto. Brasil, 2020. 


\section{Página $6,2^{\circ}$ parágrafo:}

\section{Onde se lia:}

Considerando-se a distribuição dos casos de acordo com a UF de residência, São Paulo teve a maior frequência de pacientes hospitalizados, com 1.320 (26,8\%), dos quais 1.247 (94,5\%) se recuperaram e 73 (4,5\%) evoluíram a óbito. Em seguida, vem o Amazonas com $517(10,5 \%)$ pacientes hospitalizados, dos quais $491(95,0 \%)$ se recuperaram e $26(5 \%)$ foram a óbito (Figuras 2A e 2B e Material Suplementar).

\section{Leia-se:}

Considerando-se a distribuição dos casos de acordo com a UF de residência, São Paulo teve a maior frequência de pacientes hospitalizados, com 1.320 (26,8\%), dos quais 1.247 (94,5\%) se recuperaram e 73 (4,5\%) evoluíram a óbito. Em seguida, vem o Amazonas com $517(10,5 \%)$ pacientes hospitalizados, dos quais $491(95,0 \%)$ se recuperaram e $26(5,0 \%)$ foram a óbito (Figuras 2A e 2B e Material Suplementar).

\section{Página $8,7^{\circ}$ parágrafo:}

\section{Onde se lia:}

$\mathrm{Na}$ análise bivariada, observou-se que tiveram maior risco de evoluir ao óbito os casos que apresentaram os seguintes fatores: ser do grupo etário de adolescentes $(\mathrm{RR}=1,49$; IC95\% 1,26-1,78; $<<0,001)$; ser da raça/ cor autodeclarada preta/parda (RR = 1,34; IC95\% 1,08-1,68; $<<0,008)$; ter sido classificado como SRAG-crítico (RR = 4,13; IC95\% 3,43-3,96; $\mathrm{p}<0,001)$; ter cardiopatia $(\mathrm{RR}=2,07 ; \mathrm{IC} 95 \% 1,58-2,72 ; \mathrm{p}<0,001)$, imunopatia $(\mathrm{RR}=1,74$; IC95\% 1,32-2,30; $p<0,001)$, diabetes $(R R=1,57$; IC95\% 1,08-2,30; $p=0,032)$ e neuropatia $(\mathrm{RR}=1,47 ; \mathrm{IC} 95 \% 1,09-1,97 ; \mathrm{p}=0,013)$. A asma configurou-se como fator de redução do risco para o óbito $(\mathrm{RR}=0,25 ; \mathrm{IC} 95 \% 0,10-0,62 ; \mathrm{p}=0,003$ (Tabela 2 ).

Outras variáveis relacionadas aos sinais e sintomas respiratórios foram testadas na análise bivariada, quais sejam: baixa saturação de oxigênio (RR = 4,12; IC95\% 3,33 - 5,09; p < 0,001), desconforto respiratório ( $R R=3,23$; I95\% 2,57 - 4,06; $\mathrm{p}<0,001)$, dispneia $(R R=3$; IC95\% $2,39-3,78 ; \mathrm{p}<0,001)$ e cianose $(\mathrm{RR}=2,89$; IC95\% 1,66 - 5,05; $\mathrm{p}=0,003)$ (dados não apresentados em tabela). Essas variáveis foram agrupadas no conjunto da variável SRAG-crítico para compor o melhor modelo de regressão.

\section{Leia-se:}

$\mathrm{Na}$ análise bivariada, observou-se que tiveram maior risco de evoluir ao óbito os casos que apresentaram os seguintes fatores: ser do grupo etário de adolescentes $(R R=1,49$; IC95\% 1,26-1,78; $\mathrm{p}<0,001)$; ser da raça/cor autodeclarada preta/parda $(\mathrm{RR}=1,34 ; \mathrm{IC} 95 \%$ 1,08-1,68; $\mathrm{p}=0,008)$; ter sido classificado como SRAG-crítico (RR = 4,13; IC95\% 3,43-4,97; $\mathrm{p}<0,001)$; ter cardiopatia (RR $=2,07 ; \mathrm{IC} 95 \% 1,58-2,72 ; \mathrm{p}<0,001)$, imunopatia $(\mathrm{RR}=1,74$; IC95\% 1,32-2,30; $\mathrm{p}<0,001)$, diabetes ( $R R=1,57$; IC95\% 1,08-2,30; $\mathrm{p}=0,032$ ) e neuropatia $(\mathrm{RR}=1,47 ; \mathrm{IC} 95 \%$ 1,09-1,97; $\mathrm{p}=0$,013). A asma configurou-se como fator de redução do risco para o óbito $(\mathrm{RR}=0,22$; IC95\% 0,12-0,39; $\mathrm{p}<0,001$ (Tabela 2). 
Outras variáveis relacionadas aos sinais e sintomas respiratórios foram testadas na análise bivariada, quais sejam: baixa saturação de oxigênio $(R R=4,12$; IC95\% 3,33 - 5,09; p < 0,001), desconforto respiratório $(R R=3,23$; IC95\% 2,57-4,06; $p<0,001)$, dispneia $(R R=3,0 ;$ IC95\% $2,39-3,78 ; \mathrm{p}<0,001)$ e cianose $(\mathrm{RR}=2,89$; IC95\% 1,66 - 5,05; $\mathrm{p}=0,003)$ (dados não apresentados em tabela). Essas variáveis foram agrupadas no conjunto da variável SRAG-crítico para compor o melhor modelo de regressão.

\section{Páginas 8 e $10,9^{\circ}$ parágrafo:}

\section{Onde se lia:}

A análise multivariada mostrou que permaneceram como fatores associados ao óbito pela COVID-19: pertencer ao grupo etário adolescente ( $R R=1,59 ;$ IC95\% 1,12-2,25; $\mathrm{p}=0,009)$ ter sido classificado como SRAG-critico (RR = 4,56; IC95\% 2,77-7,51; $<<0,001)$ e apresentar imunopatia $(\mathrm{RR}=2,24$; IC95\% 1,58 - 3,17; $\mathrm{p}<0,001)$. A asma permaneceu como fator associado à redução do risco para o obito $(\mathrm{RR}=2,24$; IC95\% 1,58 - 3,17; p < 0,001) (Tabela 2).

\section{Leia-se:}

A análise multivariada mostrou que permaneceram como fatores associados ao óbito pela COVID-19: pertencer ao grupo etário adolescente $(\mathrm{RRa}=1,59$; IC95\% 1,12 - 2,25; $\mathrm{p}=0,009)$, ter sido classificado como SRAG-critico $(\mathrm{RRa}=4,56$; IC95\% 2,77 - 7,51; $\mathrm{p}<0,001)$ e apresentar imunopatia ( $\mathrm{RRa}=2,24$; IC95\% 1,58-3,17; $\mathrm{p}<0,001)$. A asma permaneceu como fator associado à redução do risco para o óbito (RRa $=0,25 ; \mathrm{IC} 95 \%$ $0,10-0,62 ; \mathrm{p}=0,003$ ) (Tabela 2 ). 